\title{
Análisis del ciclo de vida de las publicaciones sobre la producción de quinua (Chenopodium quinoa Willd), a través de curvas en $\mathrm{S}$
}

\section{Life-cycle analysis of the publications in quinoa (Chenopodium quinoa Willd) production, by S-curve}

\author{
Miguel Ángel García-Parra' \\ Nubia Zoraida Plazas-Leguizamón² \\ Recibido: julio 23 de 2018 \\ Aceptado: octubre 23 de 2018
}

\section{Resumen}

El objetivo del trabajo fue estudiar el ciclo de vida de las publicaciones relacionadas con la producción de quinua (Chenopodium quinoa Willd), mediante las curvas en $\mathrm{S}$. La revisión bibliográfica se efectuó en la base de datos Scopus. El análisis de la información sistematizada en el tiempo, se hizo con curvas en $\mathrm{S}$ que fueron construidas en el programa estadístico SigmaPlot ${ }^{\oplus}$. Los resultados indican que las investigaciones se centran en las relaciones entre: Chenopodium - estrés, quinua estrés, quinua - fisiología y quinua -grasa, perfilándose como áreas estratégicas de investigación y desarrollo tecnológico. Igualmente, el estudio de la quinua presenta avances significativos en Europa y Estados Unidos, mientras que en suramérica se han desarrollado principalmente en Argentina y Chile. Lo anterior permite deducir el bajo interés científico e investigativo de países productores y exportadores de quinua, como: Bolivia, Perú, Ecuador y Colombia. Esto último se constituye en una oportunidad para que los países de origen de la quinua, establezcan líneas de investigación y programas de formación de alto nivel, encaminados al fortalecimiento de la quinua como un alimento promisorio en la búsqueda de la seguridad alimentaria.

Palabras clave: quinua, curvas en $\mathrm{S}$, proteína vegetal, seguridad alimentaria.

\begin{abstract}
The objective of the study was to study the life-cycle of publications related to quinoa (Chenopodium quinoa Willd) production, by means of in S-curves. The bibliographical revision was made in the Scopus database. The analysis of the information systematized over time was done with S-curves that were built in the SigmaPlot ${ }^{\circledast}$ statistical program. The results indicate that the research focuses on the relationships between Chenopodium - stress, quinoa - stress, quinoa - physiology and quinoa - fat, being identified as strategic areas of research and technological development.In the same way, the study of quinoa presents significant advances in Europe and the United States, while in South America they have been developed mainly in Argentina and Chile. The above allows deducing the low scientific and research interest of producing and exporting countries of quinoa, such as: Bolivia, Peru, Ecuador and Colombia. The latter is an opportunity for the countries of origin of quinoa to establish lines of research and high-level training programs, aimed at strengthening quinoa as a promising food in the search for food security.
\end{abstract}

Keywords: quinoa, S-curves, vegetable protein, food security.

1 Ingeniero Agropecuario, Estudiante de Doctorado en Ciencias Agrarias y Agroindustriales, Fundación Universitaria Juan de Castellanos, Tunja, Colombia. E-mail: mangelgarcia@jdc.edu.co

2 Ingeniera Agrónoma, Estudiante de Doctorado en Desarrollo Sostenible, Fundación Universitaria Juan de Castellanos, Tunja, Colombia.E-mail:nplazas@jdc.edu.co 


\section{Introducción}

Las investigaciones desarrolladas en torno al cultivo de la quinua (Chenopodium quinoa Willd) en diferentes partes del mundo, han permitido que esta planta logre ser objeto de estudio, debido a sus características inigualables de proteína, aminoácidos, fibras, extracto etéreo, carbohidratos y su potencialidad agroindustrial, a raíz de los contenidos de fenoles, flavonoides, betalinas y su capacidad antioxidante (Valencia et al., 2017; García-Parra et al., 2018). Estas características le han permitido a la quinua posesionarse como una de las principales estrategias alimentarias de niños, jóvenes y adultos (Chito et al., 2017).

En los años 80 y 90, países como: Estados Unidos, Italia, Irán, Egipto, Emiratos Árabes, Dinamarca y Grecia, se interesaron por buscar materiales vegetales de quinua, que lograran adaptarse a las condiciones propias de clima y suelo. Esto con el ánimo de diversificar sus alternativas agroalimentarias de origen vegetal, que les permitiera resolver problemáticas de seguridad alimentaria (Bazile et al., 2016). El interés por establecer este cultivo en zonas difíciles de suelo y clima, se debe a que ésta planta tiene la capacidad de tolerar condiciones climáticas adversas, como: heladas, salinidad y cambios fuertes de temperatura.

En este sentido, gran parte de las investigaciones científicas y las tendencias de desarrollo respecto a la quinua, se encuentran en internet y principalmente en bases de datos especializadas, como Scopus y Journal Citation Report, JCR. Esto permite el modelamiento de la información científica, con el fin de conocer el estado de desarrollo de las investigaciones en el tiempo frente a la producción de este importante pseudocereal, dando como resultado el pronóstico del ciclo de vida del tema de interés.

El ciclo de vida de una tecnología o área en particular, es el proceso de desarrollo expresado por cualquier producto o servicio durante un periodo determinado, influenciado por factores de mercado y consumo. Según Zartha y colaboradores (2017), este puede encontrarse: emergente, entrante, clave, maduro o en declive, de acuerdo con el punto de inflexión de la curva. La información así obtenida permite tomar decisiones sobre la propuesta de investigaciones para trabajos de maestría, doctorado, líneas de investigación, proyectos estratégicos o desarrollo de empresas de spin-off. Una de las principales herramientas que facilita la organización y proyección de la información a través de seguimiento del ciclo de vida, son las curvas en S (Silling \& Esmundo, 2009). Este proceso de construcción de regresiones no lineales, muestran a través de los ejes en función del tiempo y el número de publicaciones, las áreas estratégicas a abordar en el desarrollo de nuevas tecnologías (Zartha et al., 2010).

El objetivo del presente estudio fue evaluar el ciclo de vida de la producción de quinua (Chenopodium quinoa Willd), mediante el modelamiento en curvas en S, para identificar el desarrollo científico a través del tiempo en temas clave del producto. Los resultados permiten plasmar el panorama actual de las investigaciones publicadas en artículos científicos, que aportan al desarrollo de nuevos proyectos de investigación.

\section{Materiales y métodos}

En esta investigación se tomaron como variables de evaluación: estrés en quenopodiáceas, estrés en quinua, fisiología de quinua, proteína de quinua, fibra de quinua y grasa de quinua, para ser evaluados a través de curvas en $\mathrm{S}$ siguiendo la metodología propuesta por Aguilar y colaboradores (2012). Se seleccionó la base de datos Scopus, utilizando documentos publicados, como: artículos a través del tiempo organizados por año, número de publicaciones acumuladas y tabuladas en el programa Microsoft Excel ${ }^{\circledR}$. 
Tabla 1. Variables evaluadas en la base de datos Scopus.

\begin{tabular}{cc}
\hline Área de relación & Ecuación de búsqueda: Artículos \\
\hline Chenopodium y estrés & TITLE-ABS-KEY(chenopodium AND stress) \\
Quinua y estrés & TITLE-ABS-KEY(quinoa AND stress) \\
Quinua fisiología & TITLE-ABS-KEY(quinoa AND Physiology) \\
Quinua y proteína & TITLE-ABS-KEY(quinoa W/10 protein) \\
Quinua y fibra & TITLE-ABS-KEY(quinoa W/10 fiber) \\
Quinua y grasa & TITLE-ABS-KEY(quinoa W/10 fat) \\
Quinua y gluten & TITLE-ABS-KEY(quinoa AND gluten) \\
\hline
\end{tabular}

La sumatoria de los datos obtenidos de los artículos publicados para cada una de las variables, fueron ingresados en el Software Sigmaplot ${ }^{\circledR}$, con categoría de ecuación sigmoidal en tanto que fue el modelo de mejor ajuste (sigmoid-3). Luego se calcularon los puntos de inflexión para la fórmula de búsqueda, validados a través del valor Rajustado, $\mathrm{t}$ (mayor a 2 y menor a -2$), \mathrm{P}(<0.005)$, y Durbin Watson (DW). Finalmente, obtenidos los resultados, se analizaron las regresiones y los parámetros estadísticos de las investigaciones encontradas, para cada uno de los temas listados en la tabla 1.

\section{Resultados y discusión}

En la tabla 2 se presentan los países con mayor número de artículos publicados en la base de datos Scopus, para cada ecuación de búsqueda. Los países más sobresalientes fueron Europeos y Norteamericanos, indicando una desventaja para los países netamente productores de quinua; esto implica su bajo interés en la publicación de investigaciones científicas y académicas en torno a este cultivo.

Tabla 2. Países con mayor número de publicaciones (>10\%), en cada una de las ecuaciones de búsqueda, según Scopus.

\begin{tabular}{ccc}
\hline Ecuación de búsqueda & Países con mayor & Participación (\%) \\
(chenopodium AND stress) & China & 17 \\
(quinoa AND stress) & Dinamarca & 13 \\
& Estados Unidos & 10 \\
(quinoa AND Physiology) & Dinamarca & 18 \\
& Italia & 11 \\
(quinoa W/10 protein) & Chile & 10 \\
& Dinamarca & 12 \\
& Australia & 10 \\
(quinoa W/10 fiber) & Francia & 10 \\
& Estados Unidos & 13 \\
& Argentina & 11 \\
(quinoa W/10 fat) & Chile & 10
\end{tabular}




\begin{tabular}{ccc}
\hline Ecuación de búsqueda & $\begin{array}{c}\text { Países con mayor } \\
\text { número de publicaciones }\end{array}$ & Participación (\%) \\
\hline (quinoa AND gluten) & Alemania & 16 \\
& Italia & 15 \\
& Irlanda & 13 \\
& Estados Unidos & 12 \\
Brasil & 11 \\
\hline
\end{tabular}

En los siguientes apartados se presenta el análisis de las curvas en $\mathrm{S}$, número de publicaciones Vs tiempo en años, para cada una de las ecuaciones de búsqueda.

\subsection{Relación Chenopodium y estrés}

Las investigaciones en torno a la subfamilia Chenopodiaceae muestran importantes resultados, evidenciando que en 1984 se realizó la primera publicación sobre el tema en Scopus. Las publicaciones se incrementaron hasta acumular 277 artículos, con un crecimiento coordinado entre la tendencia, el número de publicaciones por año y la predicción, como se muestra en la figura 1. Además, se observó que los países con mayor número de publicaciones en la relación Chenopodium y quinua fueron: China, Dinamarca, Estados Unidos, Alemania e Italia, respectivamente, ver tabla 2.

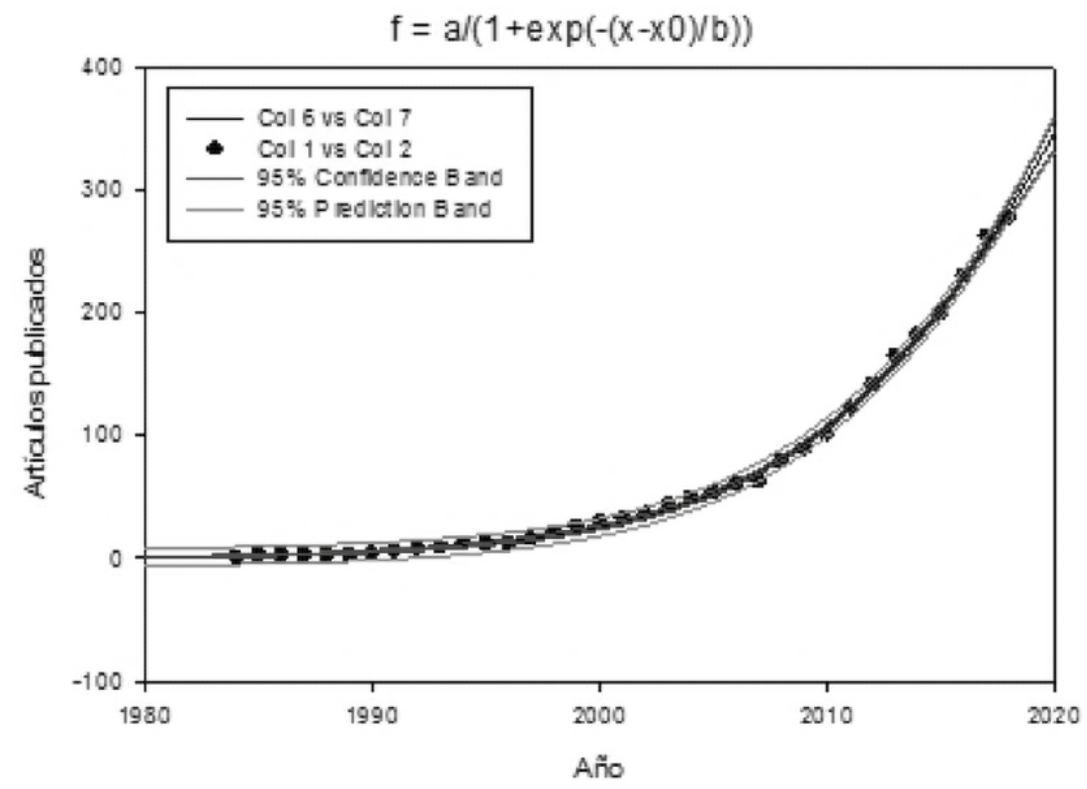

Figura 1. Curvas en $S$ para la ecuación: (chenopodium AND stress). $N=277 ; R^{2}: 0.998 ; P<0.0001 ; t>2 ; D W: 1.277$.

El ajuste del parámetro $\mathrm{R}^{2}$ de la figura 1, arroja un resultado superior al 99\%, junto con el valor t ma- yor a 2, evidenciando que el modelo Sigmoid-3 parámetros representa adecuadamente el com- 
portamiento de Chenopodium y stress en el tiempo. Esto puede explicar que las publicaciones del estrés en esta variable, se ha incrementado significativamente durante los últimos años, manteniéndose como tema clave, debido a que el punto de inflexión se encuentra en el 2022, con proyecciones crecientes para el desarrollo de investigaciones en torno a este tema.

Lo anterior concuerda con Tanveer y Shah (2017), quienes afirman que esta subfamilia de plantas se han podido cultivar en condiciones adversas de clima y suelo, aptas para la mitigación en el cambio climático, debido a que soportan el estrés generado por: la salinidad, las heladas y los cambios fuertes de temperatura. Igualmente, se identifica el adelanto de investigaciones frente a este tema, principalmente en países desarrollados que buscan alternativas de alto valor nutricional (FAO, 2015).

\subsection{Relación quinua y estrés}

La quinua se ha convertido en una de las principales fuentes alimentarias en diferentes partes del mundo, generando intereses productivos y científicos, debido a la búsqueda constante de plantas que se adapten al medio y que además sean fuentes alimentarias vegetales de alta calidad. Se valora la presencia de proteína, fibra, grasa y, en este caso, la ausencia de gluten, encontrando interés en el tema países como: Dinamarca, Italia y Chile, ver tabla 2.

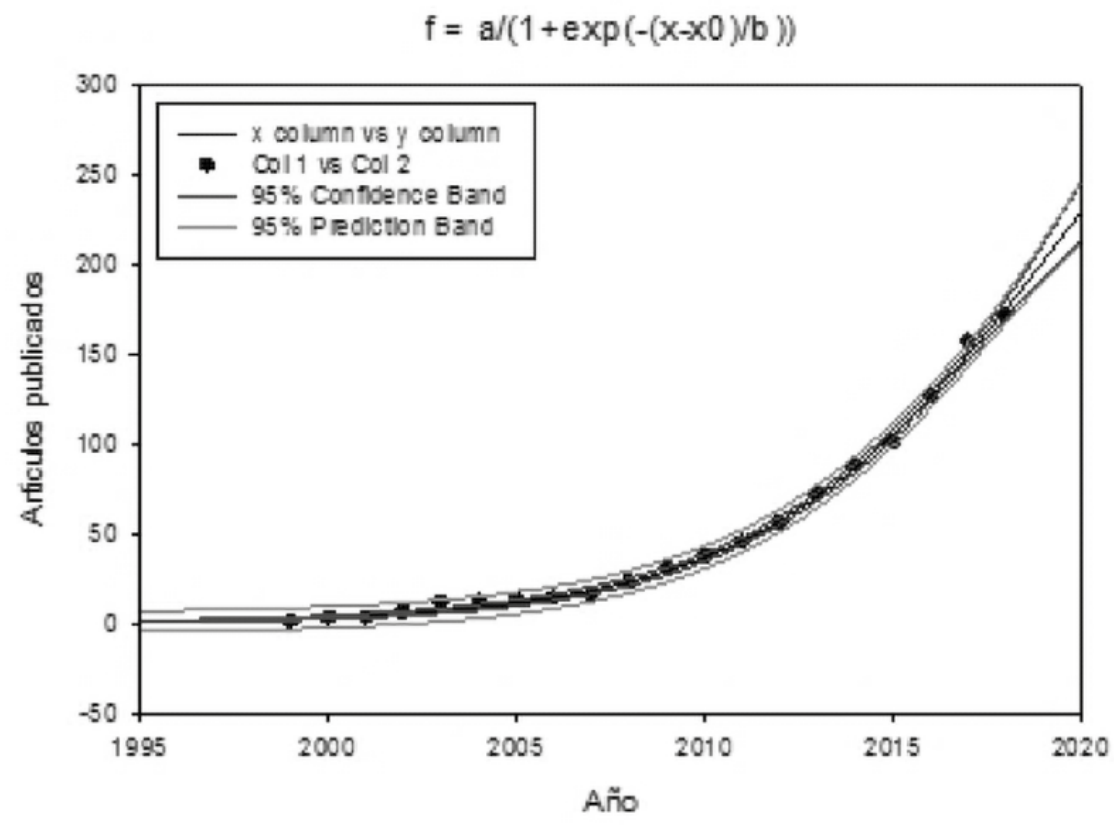

Figura 2. Curvas en S para la ecuación: (quinoa AND stress). $N=174 ; R^{2}: 0.997 ; P<0.0001 ; t>2 ; D W: 2.00029$.

El modelo que mejor se ajustó a la ecuación de búsqueda, quinoa AND stress, fue Sigmoid-3 parámetros, con un $\mathrm{R}^{2}$ ajustado superior al $99 \%$, junto con un valor t mayor que 2 y un valor $P$ menor que 0.0001 , arrojando un punto de inflexión superior al año 2018 (2020). Esto indica que el estrés en 
quinua es un tema de investigación que, según la posición del área de conocimiento propuesto por Escobar y Zartha (2017), se encuentra en etapa clave, favoreciendo el planteamiento, formulación y ejecución de propuestas en proyectos de investigación, líneas de investigación y elaboración de trabajos de maestría y doctorado.

Lo anterior es consistente con lo reportado por Melo (2016), quien afirma que el cultivo de la quinua es una estrategia de investigación de adaptabilidad a diferentes regiones estacionales; pero, en consecuencia, las plantas en etapas iniciales expresan diferentes comportamientos de estrés, de acuerdo a las condiciones de clima y suelo (Marschner, 2014).

\subsection{Relación quinua y fisiología}

La quinua es una planta que presenta características fisiológicas capaces de soportar condiciones difíciles de suelo y clima, influyentes en la expresión ecofisiológica, metabólica y genética, ver figura 3. En este caso: Dinamarca, Australia, Francia e Italia, son pioneros en la producción de artículos; sin embargo, se observó que países como Pakistán y Bolivia, una de las principales potencias productoras de quinua, reflejan un índice bajo en investigación relacionada con la fisiología del cultivo.

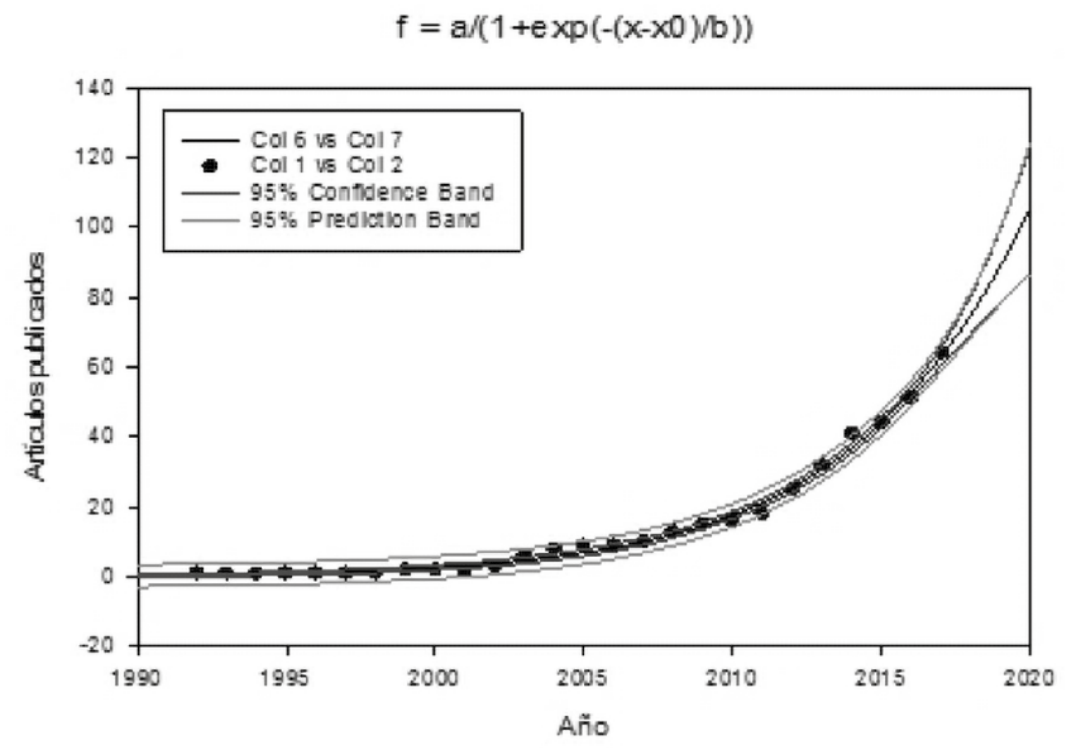

Figura 3. Curvas en S para la ecuación: (quinoa AND Physiology). $N=66 ; R^{2}: 0.9913 ; P<0.0007 ; t>2 ; D W: 1.6001$.

La ecuación de búsqueda que relaciona la fisiología de la quinua, evidenció que existen en Scopus 65 artículos, con un modelo adecuado para Sigmoid-3 parámetros y un $\mathrm{R}$ ajustado cercano al 100\%, con un punto de inflexión para el año 2019. Esto evidencia una proyección madura con potencialidad de investigación en Latinoamérica, ya que los países pioneros en investigación son principalmente Europeos.

Rojas y colaboradores (2014), afirman que gracias a la diversidad genética de Chenopodium quinoa Willd a nivel mundial, existen cerca de 16422 accesiones de quinua en los bancos de germoplasma, clasificadas en cinco grandes grupos de acuerdo a 
las zonas de producción. Esto le permite adaptarse a diferentes condiciones agronómicas, expresando respuestas fenológicas y fisiológicas, dependientes del entorno y otras condiciones.

En este sentido, la respuesta ecofisiológica de la quinua es una variable determinante en la productividad y calidad de las semillas, influyente en el potencial agroindustrial de ésta especie (Aluwi et al., 2017); en tanto que las condiciones de clima y los planes de fertilización, determinan aspectos agronómicos de la planta (García-Parra et al., 2017). Además, se encontró que Dinamarca es pionero en investigaciones relacionadas con la respuesta fisiológica de la quinua, lo que puede corresponder a la corta trayectoria que tiene éste país respecto de la expresión de este cultivo en condición estacional; algo similar a lo que sucede en países como Australia, Francia e Italia.

\subsection{Relación quinua y proteína}

Las principales fuentes proteicas son de origen animal (Camero-Escobar \& Calderón-Calderón, 2018); sin embargo, el fortalecimiento del desarrollo agroindustrial en la búsqueda de proteínas de origen vegetal se ha intensificado. En la figura 4 se observa que las investigaciones en torno a la proteína presente en las semillas de quinua, se han venido reduciendo significativamente. Se encontró que países Norteamericanos y Europeos, han desarrollado gran parte de las investigaciones existentes sobre el tema; esto como respuesta a la constante demanda alimentaria que aumenta a diario, buscando adaptabilidad de especies que presenten esta potencialidad y contengan moléculas de alto valor nutricional.

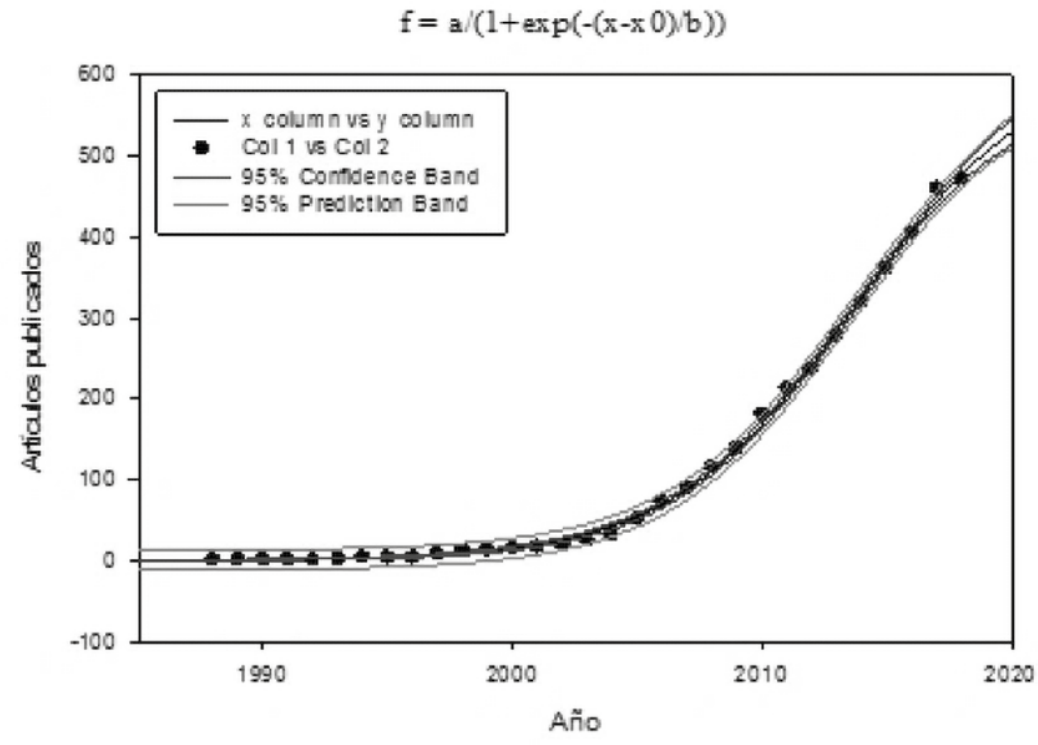

Figura 4. Curvas en $S$ para la ecuación: (quinoa $W / 10$ protein). $N=471 ; R^{2}: 0.998 ; P<0.0001 ; t>2 ; D W: 1.3369$.

Las publicaciones en artículos que relacionan la proteína de quinua, aumentaron significativamente a partir del año 2000. Esto, probablemente por la campaña para el fortalecimiento del cultivo por parte de la organización de las Naciones Unidas para la alimentación y la Agricultura, FAO, 
debido a la potencialidad de la quinua respecto a su contenido proteico y la presencia de todos los aminoácidos esenciales (Bazile et al., 2014; Peiretti et al., 2013; Navruz-Varli \& Sanlier, 2016).

El impulso de la investigación se mantuvo hasta el 2013, momento en el cual se presentó el punto de inflexión, con un $\mathrm{R}^{2}$ ajustado muy cercano a $100 \%$, t positivo mayor a 2 y con un $\mathrm{P}$ menor a 0.0001 , donde el modelo que mejor se ajustó fue sigmoid-3 parámetros. Esto permite concluir que la ecuación que relaciona proteína y quinua se encuentra en estado de declive, lo que implica el cambio del ciclo de vida en relación a la fórmula de búsqueda, generando áreas especializadas o creación de nuevos paradigmas de búsqueda (Escobar \& Zartha, 2017). Sin embargo, en países en vía de desarrollo, como Colombia, estos temas aún son de gran interés (García-Salcedo et al., 2016).

En contraste, se evidencia el interés de publicaciones por parte de Estados Unidos, Argentina, Chile e Italia, quienes aportan significativamente al conocimiento científico de los contenidos proteicos en quinua, sin influir directamente en el desarrollo primario y secundario de este producto; esto debido a que no son países productores y exportadores de quinua, como lo son Bolivia y Perú (Jäger, 2015).

\subsection{Relación entre quinua y fibra}

Las investigaciones relacionadas al contenido de fibra en el grano de quinua, han permitido fortalecer el desarrollo científico en este campo alimentario. En la figura 5 se observa que las publicaciones referentes a la ecuación quinoa AND Fiber, en la base de datos Scopus, aumentaron a partir de la década de los noventa, principalmente en países como: Estados Unidos, España, Italia, Argentina, Chile y Polonia, generando los mayores aportes respecto al contenido de este componente, de gran interés para la salud humana.

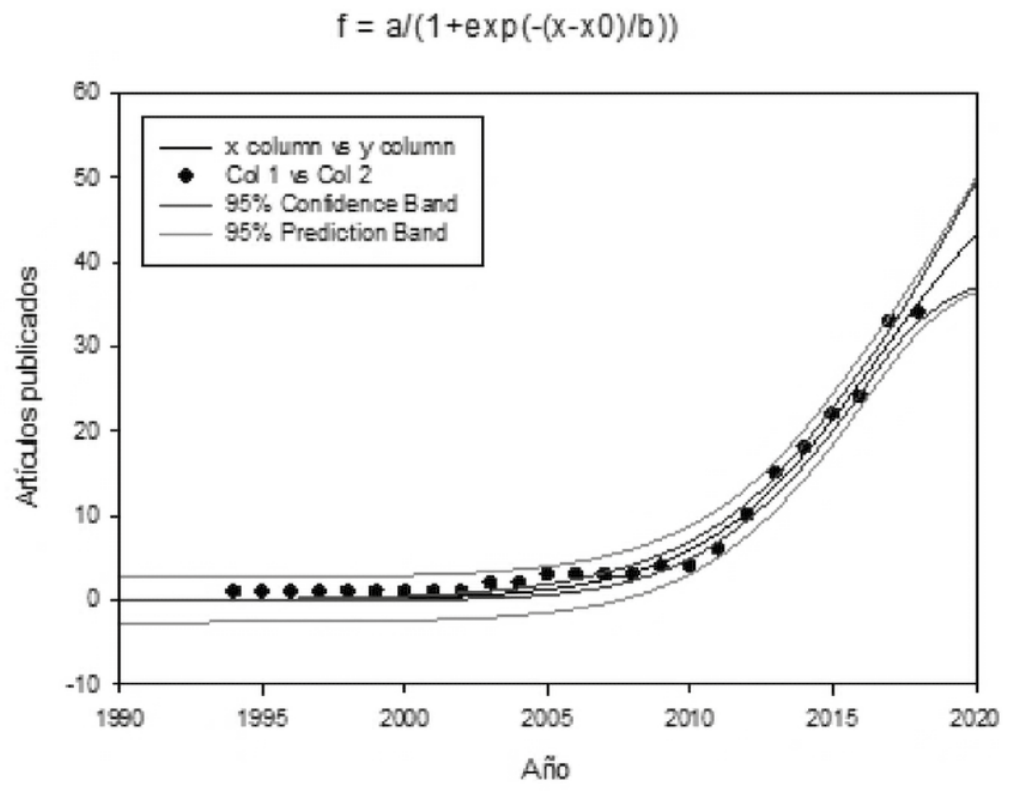

Figura 5. Curvas en $S$ para la ecuación: (quinoa $W / 10$ fiber). $N=34 ; R^{2}: 0.9846 ; P<0.0001 ; t>2 ; D W: 1.3600$. 
Los resultados evidencian que las publicaciones han venido aumentando desde el año 2010. Sin embargo, el punto de inflexión se presentó en el 2016 , con un $\mathrm{R}^{2}$ ajustado cercano al $99 \%$, con un $\mathrm{P}$ valor menor a 0.0001 , reflejando que las investigaciones encaminadas a la temática fibra de quinua, no fueron una prioridad en las características proximales de esta semilla, debido a que la fase de la curva se encuentra en declive. No obstante, dentro de los países que publicaron en esta área, según Scopus, se encuentra Colombia.

\subsection{Relación quinua y grasa}

Las investigaciones encaminadas a la presencia de ácidos grasos saturados e insaturados en las semi- llas de quinua, han tomado importancia durante los últimos años; esto ha permitido fortalecer el poder alimentario de este pseudocereal en la alimentación de niños, adultos y ancianos.

En la figura 6 se observa que las publicaciones para la ecuación de temáticas quinua y grasa han aumentado significativamente desde el 2005, encontrando 82 artículos publicados en Scopus, con un punto de inflexión para el año 2018, un $\mathrm{R}^{2}$ ajustado cercano al $100 \%$ y un $\mathrm{P}$ valor menor al 0,0001 . Se observa que predominan las investigaciones en: Estados Unidos, India y Brasil.

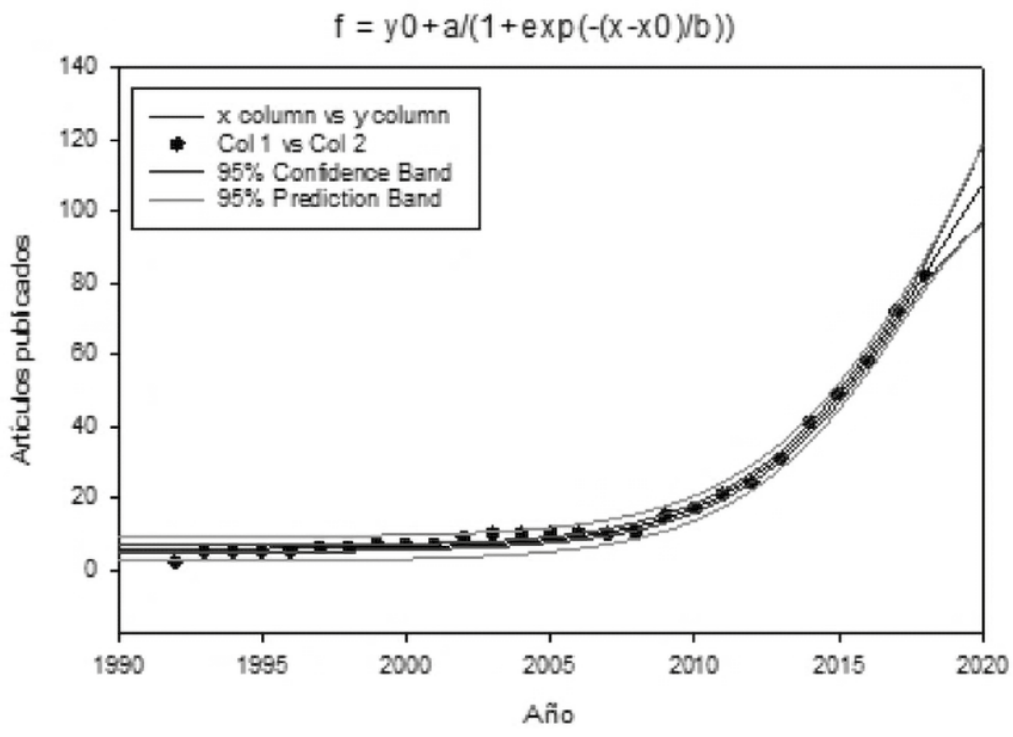

Figura 6. Curvas en $S$ para la ecuación: (quinoa $W / 10$ fat). $N=82 ; R^{2}: 0.994 ; P<0.0001 ; t>2 ; D W: 0,9466$.

El potencial alimentario y de calidad de la semilla de quinua, ha permitido que este pseudocereal se convierta en una de las principales alternativas de investigación agroindustrial, debido a que la presencia de compuestos lipídicos de alto valor alimentario permiten fortalecer el desarrollo de la extracción de grasas de origen vegetal (Vega-Gál- vez et al., 2010). Según Przybylski y colaboradores (1994), la presencia de ácidos grasos como ácido oleico, linoleico y linolenico, atribuye a la quinua otra característica alimentaria, que fortalece las investigaciones en ésta área del conocimiento alimentario. 
Además, Estados Unidos es uno de los países que ha expresado mayor interés en la investigación de la quinua con respecto a la presencia de grasas. Esto probablemente se debe a la necesidad de crear campos de investigación, con el fin de establecer nuevas fuentes lipídicas que reduzcan la problemática alimentaria de los Estados Unidos (Jumpertz et al., 2013) y que además permita adaptar este cultivo a las condiciones propias de clima y suelo (Peterson \& Murphy, 2015; García-Parra et al., 2018). Sin embargo, Colombia aparece en esta área de investigación como uno de los países con más de 10 publicaciones, lo que puede corresponder a las metas fijadas en los encuentros para el fortalecimiento de este cultivo en zonas de trópico alto andino (Jäger, 2015).

\subsection{Relación quinua y gluten}

Las investigaciones relacionadas con la presencia de gluten y su influencia en la salud humana son cada vez más frecuentes, lo que ha permitido el fortalecimiento de la búsqueda de nuevas alternativas alimentarias libres de esta proteína, que se reconoce como precursor de enfermedades celiacas (Zevallos et al., 2012). En la figura 7 se resalta la producción bibliográfica en quinua y gluten a partir del año 2000, con 151 artículos, un punto de inflexión para el 2018, P valor menor a 0.0001, en un modelo sigmoid-3 parámetros. Además, se encontró que: Alemania, Italia, Irlanda y Estados Unidos son los países que más artículos han publicado en relación a la ausencia de gluten y su viabilidad para el consumo, por parte de quienes padecen enfermedades relacionadas con el consumo de esta proteína.

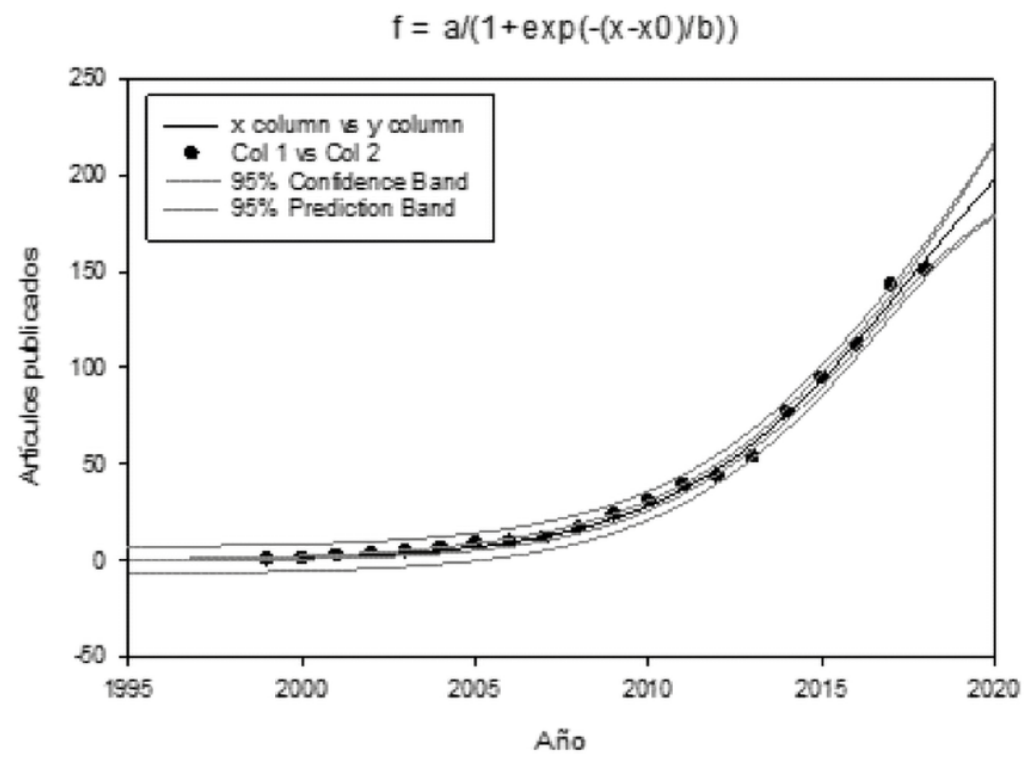

Figura 7. Curvas en S para la ecuación: (quinoa AND gluten). $N=151 ; R^{2}: 0.995 ; P<0.0001 ; t>2 ; D W: 2.1733$.

De acuerdo con los resultados, se puede afirmar que la curva en $S$ que representa la quinua y el gluten, ha mostrado una reducción significativa en el número de publicaciones por año. Esto, según Zartha y colaboradores (2014), corresponde a una tendencia en declive, como tecnología que ha pa- 
sado el punto de inflexión y por consiguiente, se generan tendencias que desligan la quinua como alimento libre de gluten, en tanto que las directrices actuales de investigación, se encaminan en la presencia de albúminas y globulinas (Janssen et al., 2017).

\section{Conclusiones}

A través del análisis del ciclo de vida de las publicaciones desarrolladas entorno al cultivo de la quinua, mediante las curvas en $\mathrm{S}$, se pudo determinar que las investigaciones publicadas en Scopus por parte de la comunidad científica, se centran actualmente en la relación entre: Chenopodium estrés, quinua - estrés, quinua - fisiología y quinua - grasa, perfilándose como áreas estratégicas de investigación y desarrollo tecnológico, según los puntos de inflexión de las curvas.

Además, las investigaciones relacionadas con el cultivo de la quinua, han avanzado fuertemente en países europeos, así como en Estados Unidos, y para el caso de Suramérica, se han desarrollado principalmente Argentina y Chile. Lo anterior permite deducir el bajo interés científico e investigativo de países productores y exportadores de quinua, como: Bolivia, Perú, Ecuador y Colombia.

La alta diversidad genética de la especie Chenopodium quinoa, facilita que los países de origen de la quinua fijen metas para la creación de líneas de investigación y programas de formación de alto nivel, maestrías y doctorados, encaminados al fortalecimiento de la quinua como un cultivo promisorio para el desarrollo en la producción primaria y secundaria. Esto último debido a la adaptabilidad de la quinua a condiciones adversas, que la identifican como cultivo óptimo para la seguridad alimentaria y de mayor resistencia a condiciones de cambio climático.

\section{Agradecimientos}

Este trabajo hace parte del proyecto: evaluación agronómica de la quinua (Chenopodium quinoa
Willd) en diferentes ambientes y su potencial agroindustrial en la zona centro de Colombia; Apoyado por la convocatoria 779-2017, de la Gobernación de Boyacá y Colciencias. Los autores agradecen al Grupo de Investigación en Agricultura, Organizaciones y Frutos, AOF, de la Fundación Universitaria Juan de Castellanos.

\section{Referencias}

Aguilar, S., Ávalos, A., Giraldo, D., Quintero, S., Zartha, J, W., \& Cortes, F. (2012). La curva en $S$ como herramienta para la medición de los ciclos de vida de productos. Journal of Technology Management \& Innovatión, 7 (1), 238-249. Recuperado de: https:// www.researchgate.net/publication/50223065_ La_Curva_en_S_como_Herramienta_para_la_Medicion_de_los_Ciclos_de_Vida_de_Productos

Aluwi, N., Murphy, K., \& Ganjyal, G. (2017). Physicochemical characterization of different varieties of quinoa. Cereal Chemistry 94 (5), 847-856. doi: https://doi.org/10.1094/CCHEM10-16-0251-R.

Bazile, D., Bertero, D., \& Nieto, C. (2014). Estado del arte de la quinua en el mundo 2013. FAO (Santiago de Chile) y CIRAD (Montpellier, Francia).

Bazile, D., Pulvento, C., Verniau, A., Al-Nusairi, M. S., Ba, D., Breidy, J., Hassan, L., Mohammed, M. I., Mambetov, O., Otambekova, M., Sepahvand, N. A., Shams, A., Souici, D., Miri, K., \& Padulosi, S. (2016). Worldwide evaluation of quinoa: preliminary results from post international year of quinoa FAO project in nine countries. Frontier Plants Science 7, 1-18. doi: https://doi.org/10.3389/fpls.2016.00850

Camero-Escobar, G., \& Calderón-Calderón, H. (2018). Vigilancia tecnológica e inteligencia competitiva para la producción de tilapia roja (Oreochromis mossambicus) en el departamento del Huila, Colombia. Revista de Investigación, Desarrollo e Innovación, 9 (1). doi: 10.19053/20278306. v9.n1.2018.8504

Chito, D. M., Ortega, R. A., Ahumada, A. F., \& Rocero, B. (2017). Quinoa (Chenopodium quinoa Willd) 
versus soja (Glycine max L Merr) en la nutrición humana. Revisión sobre características agroecológicas, de composición y tecnologías. Revista Española de Nutrición Humana y Dietética 21 (2), 184198. Recuperado de: http://renhyd.org/index.php/ renhyd/article/view/256/234

Escobar, A., \& Zartha, J. W. (2017). Application of the technology life cycle and S-curvesto the "brin drain" area of knowledge. Indian Journal of Science and Technology, 10 (43), 1-8. doi: https://doi. org/10.17485/ijst/2017/v10i43/116247

FAO. (2015). Climate change and food systems: global assesesments and implications for food security and trade. Food Agriculture Organization of the United Nations (AOF), 356. Recuperado de: http://www.fao.org/3/a-i4332e.pdf.

García-Parra, M. A., Plazas-Leguizamón, N. Z., Carvajal-Rodríguez, D. C., Ferreira-Torrado, S. K., \& Parra, J. D. (2018). Descripción de las saponinas en quinua (Chenopodium quinoa Willd) en relación con el suelo y el clima: una revisión. Informador Técnico, 82 (2), 241-249. doi: https://doi. org/10.23850/22565035.1451

García-Parra, M. A., García-Molano, J. F., \& Carvajal-Rodríguez, D. C. (2018). Evaluación del efecto de la fertilización química y orgánica en la composición bromatológica de semillas de quinua (Chenopodium quinoa Willd) en Boyacá - Colombia. Revista de investigación Agraria y Agroindustrial, 9 (2), 99-108. Recuperado de: http://hemeroteca.unad. edu.co/index.php/riaa/article/view/2282/2569

García-Parra, M., García-Molano, J., Melo, D., \& Deaquiz-Oyola, Y. (2017). Respuesta agronómica de la quinua (Chenopodium quinoa Willd) variedad dulce de Soracá a la fertilización en Ventaquemada - Boyacá. Cultura científica, JDC, 15, 66-77. Recuperado de: https://www.jdc.edu.co/revistas/index. php/Cult_cient/article/view/28/134

García-Salcedo, A., Torres-Vargas, O., \& Ariza-Calderón, H. (2016). Caracterización estructural de proteína de quinua (Chenopodium quinoa Willd) amaranto (Amaranthus caudatus L.) y Chía (Salvia hispánica L.). Agronomía Colombiana, 34 (1), 10021005. doi: https://doi.org/10.15446/agron.colomb. v34n 1 supl. 58230

Jäger, M. (2015). El cultivo de la quinua en Colombia y sus perspectivas futuras. Memorias de taller Colombia. Cali, Colombia.

Janssen, F., Pauli, A., Rombouts, I., Jansens, K., Deleu, L., \& Delcour, J. (2017). Proteins of Amaranth (Amaranthus spp.) Buckweat (Fagopyrum spp.), and quinoa (Chenopodium spp): A food science and technology perspective. Comprehensive Reviews in Food Science and Food Safety, 16, 39-57. doi: https://doi.org/10.1111/1541-4337.12240

Jumpertz, R., Venti, C., Son, D., Michaels, J., Parrinton, S., Krakoff, J., \& Votruba, S. (2013). Food label accuracy of common snack foods. Obesity (silver spring) 21 (1), 164-169. doi: https://doi. org/10.1002/oby.20185

Marschner, P. (2014). Mineral Nutrition of higher plants. Stuttgart, Alemania: Elseiver.

Melo, D. I. (2016). Studio di adattablilità dela quinoa (Chenopodium quinoa Willd) in Italia Settentrionale (Tesis doctoral). Università Cattolica Del Sacro Cuore.

Navruz-Varli, S., \& Sanlier, N. Nutritional and health benefits of quinoa (Chenopodium quinoa Willd). Journal of Cereal Science. 69, 371-376. 2016. doi: https://doi.org/10.1016/j.jcs.2016.05.004

Peiretti, P, G., Gai, F., \& Tassone, S. (2013). Fatty acid profile and nutritive value of quinoa (Chenopodium quinoa Willd) seeds and plants at different growth stages. Animal Feed Science and Technology, 183, 56-61. doi: https://doi.org/10.1016/j.anifeedsci.2013.04.012

Peterson, A., \& Murphy, K. (2015). Tolerance of lowland quinoa cultivars to Sodium Chloride and Sodium Sulfate Salinity. Crop Science, 55, 331-338. doi: https://doi.org/10.2135/cropsci2014.04.0271 
Przybylski, R., Chauhan, G., \& Eskin, N. (1994). Characterization of quinoa (Chenopodium quinoa Willd) lipids. Food Chemistry, 51 (2), 187-192. doi: https://doi.org/10.1016/03088146(94)90255-0

Rojas, W., Pinto, M., Alanoca, C., Gómez, L., León-Lobos, P., Alercia, A., Diulgeroff, S., Padulosi, S., \& Bazile, D. (2014). Capítulo 1.5. En: Bazile, D et al. Estado del arte de la quinoa en el mundo 2013: FAO (Santiago de Chile) y CIRAD (Montpellier, Francia).

Silling, M., \& Esmundo, M. (2009). Technology S-curves in renewable energy alternatives: Analisis and implications for industry and government. Energy policy, 37 (5), 1767-1781. Recuperado de: https://www.researchgate.net/ publication/46496036_Technology_S-curves_in_ renewable_energy_alternatives_Analysis_and_ implications_for_industry_and_government

Tanveer, M., \& Shah, A. N. (2017). An insight into salt stress tolerance mechanism of Chenopodium álbum. Environmental Science and Pollution Research International, 24 (19), 16531-16535. doi: https://doi.org/10.1007/s11356-017-9337-2

Valencia, Z., Cámara, F., Ccapa, K., Catacora, P., \& Quispe, F. (2017). Compuestos bioactivos y actividad antioxidante de semillas de quinua Peruana (Chenopodium quinoa Willd). Revista de la Sociedad Química Peruana, 83 (1), 16-29. Recuperado de: http://www.scielo.org.pe/scielo.php?script=sci_arttext\&pid=S1810-634X2017000100003

Vega-Gálvez, A., Miranda, M., Vergara, J., Uribe, E., Puente, L., \& Martínez, E. A. (2010). Nutrition fats and functional potential of quinoa (Chenopodium quinoa Willd.), an ancient Andean grain: A review. Journal of the Science of Food and Agriculture. 90 (15), 2541-2547. doi: https://doi.org/10.1002/ jsfa.4158

Zartha, J. W., Avalos, A. F., \& Aguilar, S. (2010). Curvas en $\mathrm{S}$, aplicación en productos innovadores del sector agroindustrial y químico Colombiano. Biotecnología en el Sector Agropecuario y Agroindustrial, 8 (2), 95-103. Recuperado: http://www.scielo. org.co/pdf/bsaa/v8n2/v8n2a13.pdf

Zartha, J. W., Zuluaga, D. F., Palacio, J.C., \& Montes, J.M. (2017). Ciclo de vida de tecnologías y curvas en $\mathrm{S}$ aplicadas en subproductos de la agroindustria piscícola. Información Tecnológica, 28 (2), 105-114. Recuperado de: https:// scielo.conicyt.cl/scielo.php?script=sci_arttex$\mathrm{t} \& \mathrm{pid}=$ S0718-07642017000200012

Zevallos, V., Allis, H., Herencia, L., \& Ciclitira, P. (2012). Variable activation of immune response by quinoa (Chenopodium quinoa Willd) prolamins in celiac disease. The American Journal of Clinical Nutrition. 96 (2), 337-344. doi: https://doi. org/10.3945/ajcn.111.030684 
